\title{
A Sound Engine for Virtual Cities
}

\author{
Shih-Han Chan, Cécile Le Prado, Stéphane Natkin, and Guillaume Tiger \\ CEDRIC, CNAM, 292 Rue Saint-Martin \\ 75003 Paris, France \\ shihhan.chan@gmail.com, cecile.leprado@free.fr, \\ stephane.natkin@cnam.fr, tiger.guillaume@gmail.com
}

\begin{abstract}
This paper is a position paper to specify and implement a generalpurpose sound engine for virtual cities. The work is motivated by the project Terra Dynamica funded by the French government. We present a state of the art of the virtual urban sound spaces emphasizing various types of virtual cities and their relationships to auditory space. We then discuss the choice of a sound engine, sound spatialization and scene description languages as ongoing works.
\end{abstract}

Keywords: Virtual City, Virtual World, Sound Engine, Soundscape.

\section{Introduction}

The purpose of this research is to study the dynamic of sound in virtual cities and to propose a sound engine solution that allows sound designers to organize virtual urban soundscapes in an interactive 3D environment. This work integrates the continuous research of the CEDRIC laboratory on sound and virtual environments.

Our work is motivated by the project Terra Dynamica, a real-time simulation of the city with avatars and non-player characters (individual characters, crowds, vehicles, flows). This project consists in both an adaptable architecture and a granular approach from individual to group. In the deliverable, real-time sound scenes are built in 3D through an open soundscape editor including procedural behavior. While sound plays different roles within different scenarios, this platform in development allows sounds to provide information, characters' locations for instance, in various fields of applications such as safety exercises and art design.

\section{State of the Art}

In the field of interactive media, considering urban representations, the audio content is closely related to the spatial organization of the city. In addition, nowadays' digital representations are often derived from soundscape studies carried on during the sixties.

Urban sound representations come from acoustic ecology and noise pollution matters. This mostly takes the form of soundmaps which consist of empirical auditory content overlaid on mapped space and, sometimes, tend to cross-media applications. Furthermore, considering the city as a designed space, its relationship to sound can be 
understood as a dynamic interaction, first through the analytic and creative paradigms of the soundscape theory, then in regard of the architectural digital acoustic tools and finally as a real-time construction within video games.

\subsection{Dynamics of Urban Auditory Environments: Soundscapes, Architectures and Video Games}

Audio, in soundscape theory, architecture and video games, is characterized by its strong relationship to spatial organization. The soundscape theory describes the auditory environment as a perspective (background, foreground and soundmarks) thus creating multidimensional dynamic objects. This perspective is echoed in architectural auditory virtual representations which provide advanced acoustic simulations calculating propagation fields (direct, diffuse, and critical). Finally, some video games offer large, real-time, interactive, 3D urban environments in which sound design strategies attempt to create a credible environment rather than a simulation.

\subsection{Usage of Sound in Virtual Cities}

We select typical applications of sound in virtual cities. Google Earth is the virtual earth representation; Second Life is also a multipurpose virtual world; Prototype is an action-adventure video game (Radical Entertainment / Activision) which uses an original dynamic sound map of New York City; Locustream is an "openmike" soundmap used for many interactive purposes; Soundwalk allows combining a localized sound recording with the real sound of the city. Figure 1 shows the use of sound in each application.

\begin{tabular}{|c|c|c|c|c|c|}
\hline $\mathrm{Ml}$ & GOOGLE EARTH & SECOND LIFE & PROTOTYPE & SOUND WALK.COM & LOCUS STREAM \\
\hline IMMERSION & Static recordings & $\begin{array}{l}\text { Public spaces, sound art } \\
\text { works and private } \\
\text { spaces }\end{array}$ & $\begin{array}{l}\text { Music, surround } \\
\text { sound, realtime } \\
\text { spatialization }\end{array}$ & $\begin{array}{l}\text { Ambient sound, read } \\
\text { text }\end{array}$ & $\begin{array}{l}\text { Realtime audio } \\
\text { streams }\end{array}$ \\
\hline NAVIGATION HELP & & & 3D sound & Audio guide & \\
\hline INFORMATION & $\begin{array}{l}\text { Relative to listenable } \\
\text { audio files }\end{array}$ & $\begin{array}{l}\text { Positioning objects (3D } \\
\text { sound), relative to audio } \\
\text { content }\end{array}$ & $\begin{array}{l}\text { Character behav- } \\
\text { iors, density / } \\
\text { proximity of agents }\end{array}$ & $\begin{array}{l}\text { Historical and } \\
\text { culture information, } \\
\text { localization }\end{array}$ & $\begin{array}{l}\text { Relative to audio } \\
\text { stream broadcasts }\end{array}$ \\
\hline SOCIALIZATION & & $\begin{array}{l}\text { Concerts, adding sounds } \\
\text { to avatars, private audio } \\
\text { broadcasts }\end{array}$ & & & User community \\
\hline STORYTELLING & & & $\begin{array}{l}\text { Interactive music, } \\
\text { dialogues }\end{array}$ & $\begin{array}{l}\text { Sound path divided } \\
\text { into sequences }\end{array}$ & \\
\hline REUSE OF DATA & & & & & $\begin{array}{l}\text { Utilization of streams } \\
\text { as sources }\end{array}$ \\
\hline
\end{tabular}

Fig. 1. Intersection of different types of virtual urban media and sound functions (red text shows functions for which users may bring content)

In all applications, the construction in layers reminds of the classical theory of soundscapes: background, foreground, and soundmarks. The dynamic organization of the soundscape is according to the needs created by the gameplay or rules of the media. 


\section{Conclusion}

Most of sound game engines have a three level architecture. The lower level includes sound synthesizers and real-time filters that can compose and be applied to a sound stream which asks for a balance between procedural sound design and wavetable playback. We choose Collada as the formal representation of the virtual space on which the intermediate level of the audio architecture relies. The definition of a sound extension of Collada is being made based on the scene language of MPEG4. The design of an efficient high-level editor is the core of our project, and is based on the analyses of several experiments and usages in the Terra Dynamica project.

We conduct various urban recordings to implement an interactive soundmap as the preliminary work. On top of that, the spectral analysis of the recordings provides data about the layers composing the urban soundscape. In our first experiment, Fmod is the lower level tool integrated with the $3 \mathrm{D}$ rendering engine - Unity3D. We will next explore other possible combinations of various sound engines (e.g Wwise, Playall) and rendering engines (e.g Ogre) with the integration of Collada sound scene.

Considering the large number of audio sources in urban virtual environments, spatialization problematic will be included in our future works as it may bring solutions regarding level of detail, source clustering and reverberation.

While the project Terra Dynamica requests audio uses within five main fields of applications (game and art, urban design and architecture, safety analysis, city transportation, proximity services), it will also provide us some visual data and basic requirements (e.g. path finding) from which we will be able to define more precisely our sound engine architecture.

\section{References}

1. Collins, K.: Game Sound. MIT Press, Cambridge (2008)

2. Wrightson, K.: An introduction to acoustic ecology. Soundscape the Journal of acoustic ecology 1(1) (2000)

3. Funkhouser, T., Tsingos, N.: Sounds Good to Me! In: Computational Sound for Graphics, Virtual Reality, and Interactive Systems. In: SIGGRAPH 2002 Course Notes (2002)

4. Veneri, O.: Architecture d'un intergiciel pour la creation sonore dans les jeux video. Phd thesis, CNAM (2009)

5. Scheirer, E., Lee, Y., Yang, J.: Synthetic Audio and SNHC Audio in MPEG-4. In: Puri, A., Chen, T. (eds.) Advances in Multimedia: Signals, Standards, and Networks. Marcel Dekker, New York (2000) 